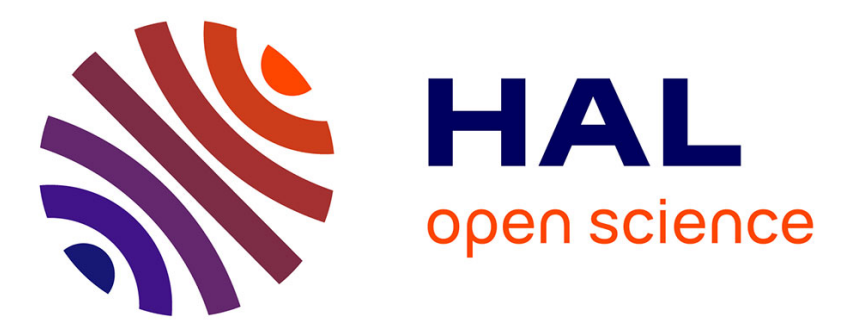

\title{
Pulse X-Ray Diffraction Study of Shock-Compressed $\mathrm{NaCl}$
}

\author{
E. Zaretsky
}

\section{To cite this version:}

E. Zaretsky. Pulse X-Ray Diffraction Study of Shock-Compressed NaCl. Journal de Physique IV Proceedings, 1997, 07 (C3), pp.C3-329-C3-334. 10.1051/jp4:1997358 . jpa-00255515

\section{HAL Id: jpa-00255515 https://hal.science/jpa-00255515}

Submitted on 1 Jan 1997

HAL is a multi-disciplinary open access archive for the deposit and dissemination of scientific research documents, whether they are published or not. The documents may come from teaching and research institutions in France or abroad, or from public or private research centers.
L'archive ouverte pluridisciplinaire HAL, est destinée au dépôt et à la diffusion de documents scientifiques de niveau recherche, publiés ou non, émanant des établissements d'enseignement et de recherche français ou étrangers, des laboratoires publics ou privés. 


\title{
Pulse X-Ray Diffraction Study of Shock-Compressed NaCl
}

\author{
E. Zaretsky
}

Pearlstone Center of Aeronautical Engineering Studies, Department of Mechanical Engineering,
Ben-Gurion University of the Negev P.O.Box 653, Beer-Sheva 84105, Israel

\begin{abstract}
Results of X-ray diffraction study of shock-compressed $\mathrm{NaCl}$ single crystals of $<100\rangle$ orientation are suggested. A new geometry of X-ray diffraction survey specially developed for studies of a single crystal sámple in the monochromatic radiation was employed to obtain a multi-peak diffraction pattern allowing the complete determination of the components of the crystal strain tensor. During the compression of the crystals their state was continuously monitored by velocity interferometer. The diffraction patterns obtained at the different regions of the plastic wave front evident that the crystal is able to maintain the state of unidimensional compression up to the strain level of about 0.06 .
\end{abstract}

Résumé : La compression par choc d'un cristal $\mathrm{NaCl}$ dans la direction < $100>$ est étudiée a l'aide de la diffraction des rayons $\mathrm{X}$. Une géométrie nouvelle d'analyse de diffraction à rayons $\mathrm{X}$ développée spécialement pour l'étude d'échantillon monocristallins soumise à un rayonnement monochrome est employée afin d'obtenir un motif à diffraction multi-crête permertant de déterminer complètement les composantes du tenseur de tension du cristal. L'état du cristal est contrôlé continuellement par un interféromètre de vitesse. Les raies de diftraction obtenues pour des régions différentes du front d'onde plastique montrent que le cristal est capable de supporter l'état de compression monodimensionnelle jusquà un niveau de tension d'environ 0,06 .

\section{INTRODUCTION}

Attempts to obtain a diffraction pattern containing a microscopic information about the strain state of the material with shock were done by a variety of the research groups during two last decades (e.g. Johnson et al. [1], Egorov et al. [2], Kondo et al. [3], Jamet and Bauer [4], Miller and Schulte [5], Zaretsky et al. [6], Wark et al. [7], Whitlock and Wark [8]). In all of these works the pulse X-ray diffraction patterns were obtained from a shock-compressed single crystals. The only exception is the work of Zaretsky et al. [6], but a strong texture of the studied aluminum made the material initial state close to that of single crystal. The pulse X-ray sources existing at present, have the intensity of the radiation insufficient to obtain diffraction pattern from polycrystalline sample. This, together with the conditions of one-dimensional shock loading of the single crystal sample, forced the researches to use the diffraction survey geometry which is usually used for obtaining diffraction pattern of monochromatic $\mathrm{X}$-ray radiation from plane polycrystalline sample, so-called Bragg-Brentano scheme. In this case the vector of the direction of the shock wave propagation $\mathbf{x}$ belongs to the plane containing the incident and the reflected $\mathrm{X}$-ray beams together with the normal vector of the crystalline planes $\mathbf{n}$ normal to $\mathbf{x}$. The lattice compression may be easily found by differentiating the Bragg's formula

$$
2 c_{200} \sin \theta_{210}=\lambda
$$

where $d_{200}$ is the (200)-planes spacing, and $\lambda$ is the wavelength of the characteristic (monochromatic in this case) radiation of the $X$-ray source, $\theta$ is the glide angle of the incident $X$-ray beam. The (200) planes are choosen here as having the strongest reflections in the case of usually studied single crystals with rocksalt structure ( $\mathrm{NaCl}, \mathrm{LiF}, \mathrm{KCl}$ ). The Eq. (1) gives for the lattice strain (for the case of small angles $\theta$ )

$$
\varepsilon_{x}=\frac{\delta a}{a}=\frac{\delta d_{200)}}{d_{200}}=-\frac{\delta \theta_{200}}{\theta_{200}},
$$

where $a$ is the lattice parameter. It is clear, that use of the Bragg-Brentano scheme in the study of shockcompressed single crystals allows to ohtain the information about the lattice strain in the direction of the 
unidimensional ( $\varepsilon_{\mathrm{x}} \neq 0, \quad \varepsilon_{\mathrm{y}}=\varepsilon_{\mathrm{z}}=0$ ) lattice compression only. It is known, however, that just behind the shock front the relaxation of the strong shear stress, developed by the unidimensional compression, lakes place. This results in the development, behind the shock front, of the material state close to the hydrostatic compression $\left(\varepsilon_{\mathrm{x}} \approx \varepsilon_{\mathrm{y}}=\varepsilon_{\mathrm{z}}\right.$ ). Thus, it is very attractive to try to obtain the complete information about the strain state of the compressed material.

The purpose of the present work is to develope a new scheme of the X-ray diffraction survey of shock-compressed single crystal which allows to obtain a diffraction pattern containing the information about crystal strains in different directions; not only in the direction of the shock compression. This scheme has been for study of shock-compressed sodium chloride single crystal whose transient behavior was continuosly monitored by velocity interferometer (VISAR, Barker and Hollenbach [9]).

\section{MULTI-PEAK X-RAY DIFFRACTION PATTERN IN SHOCK WAVE EXPERIMENTS}

It is obvious that the reflection from (200) planes remains unchangeable when the crystal is rotated around its axis parallel the [200] direction. The Bragg's equation (1) may be rewritten for reflection from arbitaly: crystalline plane (hkl)

$$
2 d_{\mathrm{hkl}} \sin \theta_{\mathrm{hkl}}=\lambda \text {. }
$$

Taking into account that for crystals of cubic symmetry (to which the rocksalt-type lattice belongs) the plane spacing $d_{\mathrm{hkl}}$ is related to the lattice period $a, d_{\mathrm{hkl}}=a / \sqrt{\mathrm{h}^{2}+\mathrm{k}^{2}+\mathrm{l}^{2}}$, the equations (1) and (i) may be rewritten

$$
\sin \theta_{\mathrm{hkl}}=\sin \theta_{\mathrm{o}} \frac{\sqrt{\mathrm{h}^{2}+\mathrm{k}^{2}+\mathrm{l}^{2}}}{2},
$$

where $\sin \theta_{200}=\lambda / a=\sin \theta_{0}$. Since the plane (200) is normal to the vector [200], the rotation of the crystal on angle $\varphi$ around axis [200] should result in arrival of the plane (hkl) to the position when Bragg's conditions (3) are satisfied. When the sample surface is parallel to the crystal (200) plane the angle $\theta$ between the plane and the vector $\mathbf{q}$ remains unchanged for any rotation angle $\varphi$. The same is correct for the projection $\mathbf{q}_{\mathrm{o}}$ of $\mathbf{q}$ on the (200) plane. In such case $\mathbf{q}=q_{\mathrm{o}}\left[-\bar{i}\left(\cos \theta_{\mathrm{o}}\right)-\bar{j}\left(\sin \theta_{0}\right)+\bar{k} \cdot 0\right]$ and its absolute vallic is $q_{o}=1$.

When the crystal is rotated around its [200] (the same as [h00]) axis the Miller indexes of the plane (hkl) will be changed according the equations:

$$
\mathrm{k}^{\prime}=\mathrm{k} \sin \varphi+\mathrm{l} \cos \varphi, \quad \mathrm{l}^{\prime}=\mathrm{l} \cos \varphi-\mathrm{k} \sin \varphi
$$

This, together with the condition $\left[\mathrm{hk}^{\prime} \mathrm{l}^{\prime}\right] \cdot \mathbf{q}=-\left[\mathrm{hk}^{\prime} \mathrm{l}^{\prime}\right] \cdot \mathbf{q}^{\prime}$ of the change of the sign of the component $\mathrm{q}_{200}^{\prime}$ of the reflected beam $\mathbf{q}^{\prime}$ parallel to the [200] direction and the condition (3a) yields for the crystal rotation on the angle $\varphi$ the Bragg's condition of the appearance of the reflection from the (hkl) plane

$$
\frac{\tan \theta_{0}}{2}=\frac{h \tan \theta_{0}-(1 \sin \varphi+k \cos \varphi)}{h^{2}+k^{2}+l^{2}} .
$$

For the film which is located at the distance $L_{v}$ from the sample axis and whose plane is normal to the plane of primary beam $\mathbf{q}$ and the crystal sample axis [200] the position of the reflection from the (hkl) plane may be found from the condition of the invariability of the sign of the glide \{along the (hk'l') plane\} component of the vector $\mathbf{q},[h k l] \times \mathbf{q}=\left[h^{\prime} l^{\prime}\right] \times \mathbf{q}^{\prime}$, and from the equation of film plane, $y=L_{n}$ :

$$
x=-L_{0} \tan \theta_{0} \frac{(1-\mathrm{h})}{1+\tan \theta_{0}(1 \sin \varphi+k \cos \varphi)}, z=L_{0} \tan \theta_{0} \frac{(1 \cos \varphi-k \sin \varphi)}{1+\tan \theta_{0}(1 \sin \varphi+k \cos \varphi)}
$$


where angle $\varphi$ should be obtained from the Bragg's condition (5) of the reflection from the given plane family (hkl).

The idea of the above scheme is close to the idea of the rotation method (e.g., Warren [10]). The scheme allows to obtain multiple-peak diffraction pattern from the single crystal radiated by the monochromatic X-rays. The rotation angle $\varphi$ for which at least two reflections exist, the reflection from the (200) plane plus a reflection from any other plane (hkl), always may be found. Accounting in that in the shock X-ray diffraction experiments the $\mathrm{Mo}_{\mathrm{K}}$ radiation is usually used and the crystal plane studied in such experiments are the crystal planes with comparatively low Miller indexes $h, k$ and l, i.e. the value of the $\tan \theta_{0} \approx \sin \theta_{0} \approx \theta_{0}$ is small, the Eqs.(6) may be simplified (with the accuracy of the first order on $\tan \theta_{0}$ ):

$$
x=-L_{0}(1-\mathrm{h}) \tan \theta_{0}, \quad z=L_{0}(1 \cos \varphi-\mathrm{k} \sin \varphi) \tan \theta_{0} .
$$

When the single crystal undergoes a shock deformation according to the scheme shown in Fig. 1 the $\mathrm{X}$-ray diffraction pattern changes. These changes may occur as a diffraction spot shift, as a broadening of the diffraction peaks (including asymmetrical broadening of the peaks), as the change of the peaks intensity.

For a crystal lattice having the rhombohedral symmetry the lattice strains $\varepsilon_{x x}$, $\varepsilon_{y y}$ and $\varepsilon_{z z}$ may be related to the variation of the indexes $h, k$ and $l$ :

$$
\varepsilon_{\mathrm{xx}}=\frac{\delta d_{\mathrm{h} 00}}{d_{\mathrm{h} 00}}=-\frac{\delta \mathrm{h}}{\mathrm{h}}, \varepsilon_{\mathrm{yy}}=\frac{\delta d_{0 \mathrm{k} 0}}{d_{0 \mathrm{k} 0}}=-\frac{\delta \mathrm{k}}{\mathrm{k}}, \varepsilon_{\mathrm{zz}}=\frac{\delta d_{001}}{d_{001}}=-\frac{\delta \mathrm{l}}{\mathrm{l}}
$$

The Eqs. (8) are useful for the application of the suggested diffraction scheme for a study of the strain state of the crystal, containing shock. In all the previous pulse X-ray diffraction studies (see References) the changes in the diffraction pattern of the shocked material was considered as the changes angle of the reflected beam $\theta_{0}$. Immediately after that the relation of type (2), which is correct for rigorous cubic symmetry only, was used to determine the compression of the material, which symmetry, under this compression, is not cubic. The suggested idea is not to consider the relation between the change of the angle $\theta_{\mathrm{o}}$, which stays constant during the experiment, as well as the angle of the crystal rotation $\varphi$. According the Eqs. (8) the initial cubic lattice is assumed to be unchanged while spatial grid of the points (hkl) undergoes the deformation. The Eqs. (6) allow to relate the initial position of the diffraction spot (peak) $\left(x_{\mathrm{i}}, z_{\mathrm{j}}\right)$ with its position in the pattern corresponding to the shocked material $\left(x_{\mathrm{j}}+\delta x, z_{\mathrm{i}}+\delta z\right)$ :

$$
\begin{aligned}
& \delta x=-\left[\frac{\partial x}{\partial \mathrm{h}} \mathrm{h} \varepsilon_{\mathrm{xx}}+\frac{\partial x}{\partial \mathrm{k}} \mathrm{k} \varepsilon_{\mathrm{yy}}+\frac{\partial x}{\partial \mathrm{l}} \mathrm{l} \varepsilon_{\mathrm{zz}}\right] \\
& \delta z=-\left[\frac{\partial z}{\partial \mathrm{h}} \mathrm{h} \varepsilon_{\mathrm{xx}}+\frac{\partial z}{\partial \mathrm{k}} \mathrm{k} \varepsilon_{\mathrm{yy}}+\frac{\partial z}{\partial \mathrm{l}} \mathrm{l} \varepsilon_{\mathrm{zz}}\right]
\end{aligned}
$$

Calculating the partial derivatives from (6) one has for the relations between the peak shifts and the lattice strain (the terms with $\tan ^{2} \theta_{0}$ are neglected)

for the reflection 200 :

$\frac{\delta x_{200}}{x_{200}}=-\varepsilon_{\mathrm{xx}} ; \delta z_{200}=0$ for the reflection 220 :

$\frac{\delta x_{220}}{x_{220}}=-\varepsilon_{\mathrm{xx}} ; \quad \frac{\delta z_{220}}{z_{220}}=-\varepsilon_{\mathrm{yy}}$

The use of Eq. (10) makes it possible the complete determination of the crystal strain state on the basis of the multi-peak X-ray diffraction pattern.

\section{EXPERIMENTAL}

Three $\mathrm{NaCl}$ single crystals, $\mathrm{N} 1$ to $\mathrm{N} 3$, of 5 -mm thickness backed with polycrystalline pyrolitic graphite 0.5 mm window were shocked by impactors, the disks of aluminum alloy 6061-T6, accelerated in the 3 -m barrel of $25-\mathrm{mm}$ pneumatic gun up to velocities about $285 \mathrm{~m} / \mathrm{sec}$. The scheme of the experimental setup with the diagnostic tools is shown in Fig. 1. Velocity and trigger (lapped together with the sample front 
surface) electrical pins were mounted in the sample body. In different experiments the 30 -nsec pulse X-rill source (based on Scandiflash $300 \mathrm{X}$-ray pulse system having pumped tube with 1 -mm focus changeialli: Mo anode) was triggered at the different instances after arrival of the shock wave at the NaCl-graphitio interface. The X-ray diffraction pattern was captured by the record system containing the 75 -mmm scintillating screen, fiber optic cone Hamamatsu V 1366P Image Intensifier and Hamamatsu C4346-0I CCD camera connected with IBM486 computer via Data Translation Frame Grabber EZ-55. The instance of the $\mathrm{X}$-ray appearance was detected by $14-\mathrm{m}$ optic fiber (10-nsec rise time scintillator) coiled around the X-rill: tube and coupled with 2.5-nsec R647 Hamamatsu photomultiplier tube. All the experiments were accompanied with continuous monitoring of the velocity of the free surface of the graphite window by th: VISAR. To enhanse the window surface reflectivity the $10-\mu$ aluminum foil was glued (glue layer of ahout $4 \mu$ ) on the surface. In parallel the quantitative information about the path of the free anvil surface and thit sample anvil interface was obtained. This information is necessary for estimation of the possible shock. induced departure of the studied sample from the initially fitted crystal position corresponding the Bratg: condition. The impactor-sample misalignment does not exceed 0.2 milliradian in all shots.

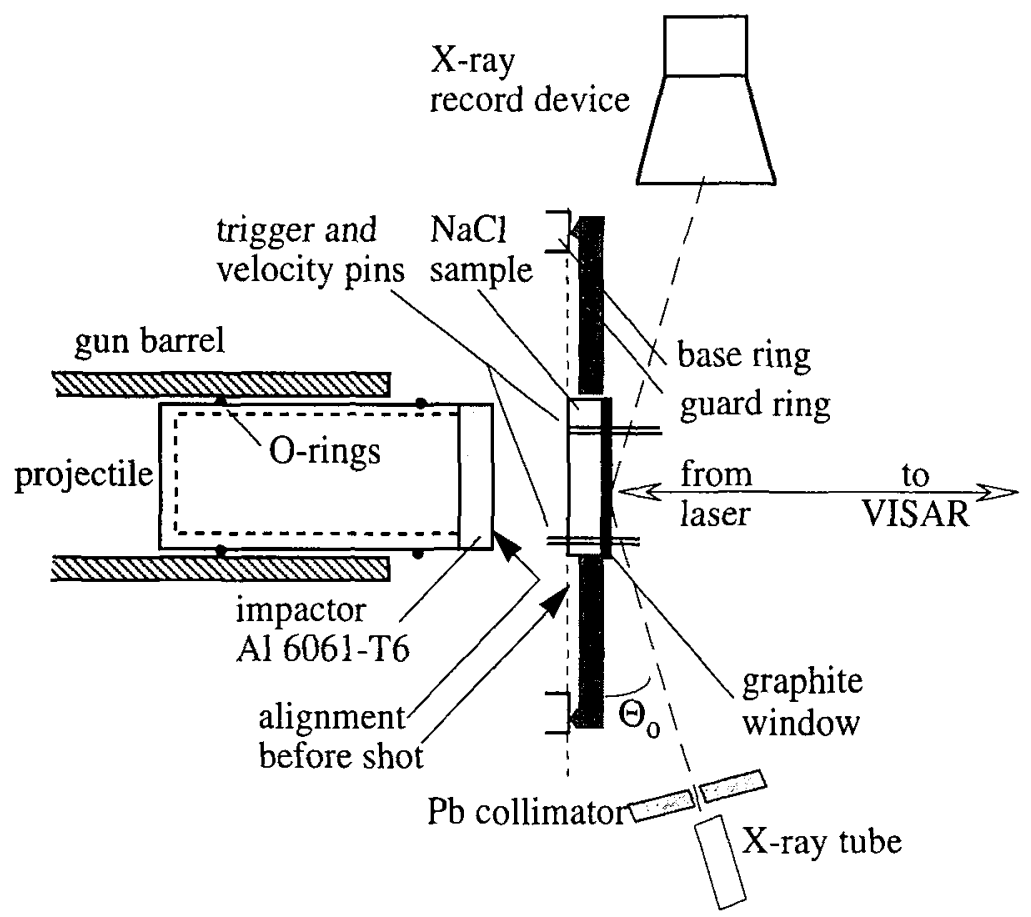

Figure 1: Scheme of the experimental setup for pulse X-ray diffraction measurements accompanied by continuous monituring of the velocity of the free surface of the graphite window.

\section{RESULTS AND DISCUSSION}

The results of the VISAR measurements, the window free surface velocity profile, in one of these experiments are shown in Fig. 2. Since the impact conditions were close in all three shots the instances of the appearance of the $X$-ray radiation in all the experiments are shown in Fig. 2. As is clear from Fig. 2 thill for any time instance at the velocity profile front the path of the sample free surface does not exceed 100 micron and for any shot the path of the sample-window interface cannot exceed several microns. Thus lor the experiments described below the influence of the interface motion on the diffraction pattern (possible variation of the Bragg's condition) should be neglected. The diffraction patterns obtained at the shot $\times 2$ are shown in Fig. 3 .

Analysis of the change of the intensity distribution of the patterns allows to obtain informutin: about the peaks shifts and the strain state of the crystal. 


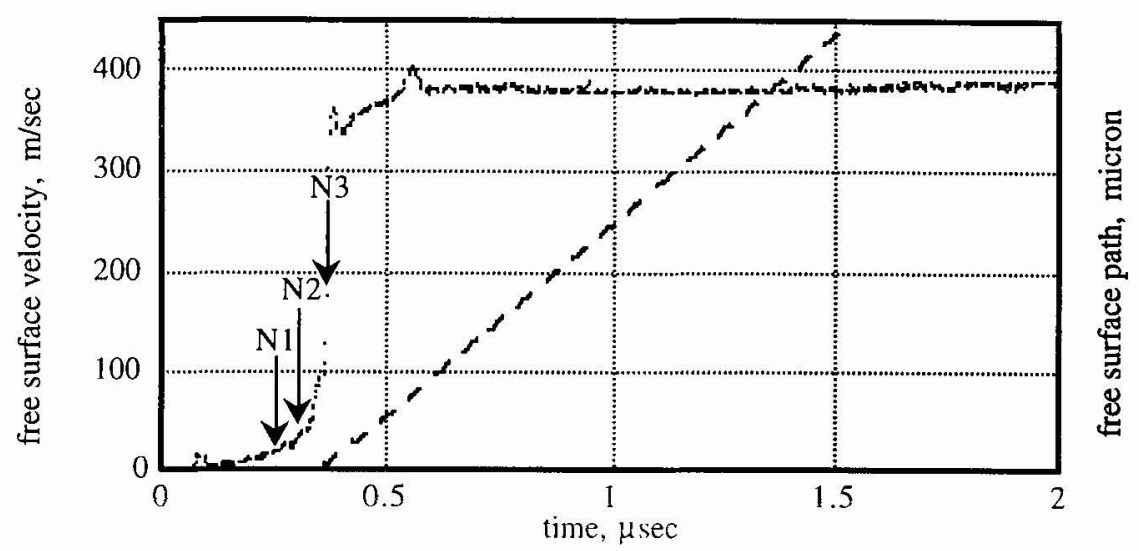

Figure 2: VISAR measurements of the free surface velocity of the sample-window-foil assembly. Impact velocity is 286 $\mathrm{m} / \mathrm{sec}$ (shot No 2). The points are the VISAR record of the velocily. The dashed line is the window free surface path. The surface velocity peaks are possible due the quality of the sample-anvil bonding (according -200 -nsec period of the peaks appearance). The arrows shows the time instances of the $\mathrm{X}$-ray flash appearance in the corresponding shot.
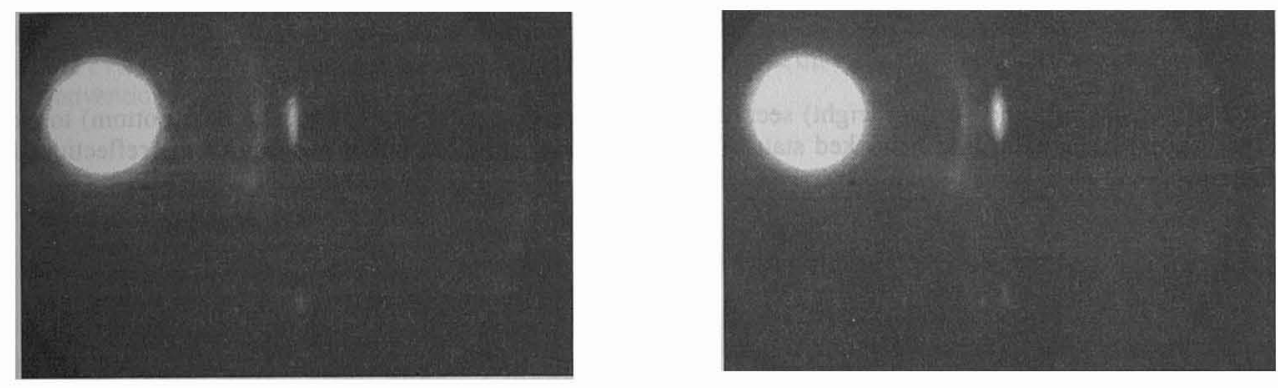

Figure 3: X-ray diffraction patterns obtained from $\mathrm{NaCl}$ single crystal beforc (left) and during (right) the shot 2. Large spots are the primary beam traces. Right spots at the horizontal line passing the PB trace are the reflections from (200) planes. Spots at the bottom part of the patterns are the reflection from (220) planes. Ark-like reflections from polyerystalline graphite are also seen.

The changes of the intensity profiles for the three shots are shown in the Fig. 4. Horizontal profiles correspond the intensity distribution along the straight line between the centers of the primary beam trace and the (200)spot.The vertical profiles correspond the distribution along the (200) - (220) line. As is clear from Fig. 4 the shots $\mathrm{N} 1$ and $N 2$ result both in the shift of the peaks in the horizontal direction and in the increase of the intensity of the peaks. The latter may be attributed to the suppress of the extinction by the strained crystal which became less perfect. At the shot N 3 the drop of the intensities of both (200) and (220) peaks is evident. In this case the crystal is far from Bragg's conditions because of the decrease of the lattice plane spacing in the direction of the wave propagation. The strain values corresponding to the peak shifts in the left part of Fig. 4 are $\varepsilon_{\mathrm{xx}}=0.006 \pm 0.001$ for shot $\mathrm{N} 1, \varepsilon_{\mathrm{xx}}=0.013 \pm 0.001$ for shot $\mathrm{N} 2$ and $\varepsilon_{x x}=0.062 \pm 0.001$ for shot $N 3$. The latter value agrees well with stain value determined from known dependence of the shock velocity $\mathrm{D}$ on the particle velocity $\mathrm{U}$ in $\mathrm{NaCl}, \mathrm{D}=3440+1.45 \mathrm{U}$, [4], and measured velocity of the free surface of the graphite window, $w=380 \mathrm{~m} / \mathrm{sec}$ (see. Fig. 2). The latter corresponds to the particle velocity in $\mathrm{NaCl}$ equal to $\mathrm{U}=230 \mathrm{~m} / \mathrm{sec}$, to the shock pressure amplitude in $\mathrm{NaCl} \mathrm{P}=1.75 \mathrm{GPa}$ and to the unidimensional compression $\varepsilon=0.061$. Note that no sign of the shift of (220) peaks in vertical direction cannot be found in the right part of Fig. 4. It means (see Eq. 10) that the crystal strain in the direction normal to the direction of the wave propagation is equal to zero. The coincidence of the strain value obtained from the changes of the diffraction pattern with the strain obtained from the hydrodynamic data with assumtion of unidimensional compression confirms that the material is able to maintain the state of 1-D strain under intensive plastic deformation. The plastic deformation of the rocksalt lattice should be obligatory accompanied by the dislocation glide in the $\{110\}$ planes of the lattice. This glide, seems, does not result in the change of the spacing of the glide planes in the direction normal to the direction of the wave propagation in the sample. 


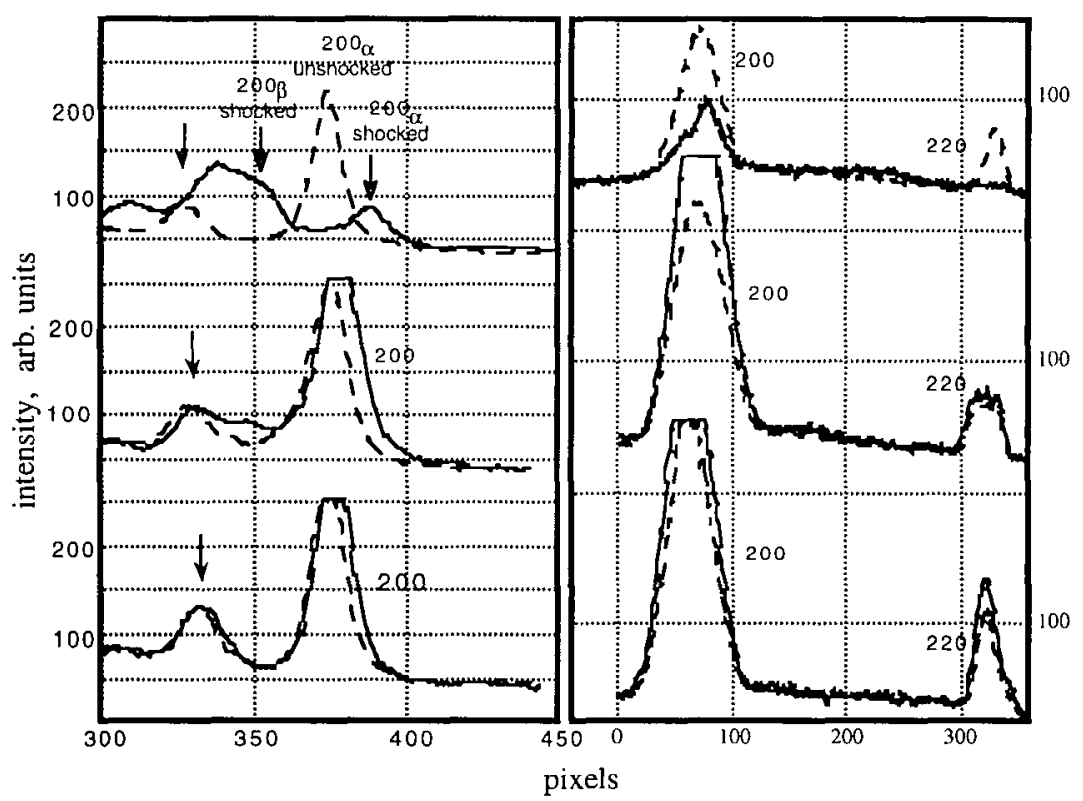

Figure 4: Horizontal (left) and vertical (right) sections of the diffraction patterns of the shots Nol(bottom) to No3 (top) Dashed line -initial state, solid line - shocked state. The filled arrows show the initial position of the reflections from the graphite anvil.

\section{Acknowledgement}

The work is supported by the United States-Israel Bi-National Science Foundation under grant No 92300316.

\section{References}

[1] Johnson Q., A.Mitchell, R.N.Keeler, and L.Evans, Phys. Rev. Lett, 25, (1970) 1099

[2] Egorov L.A., E.V.Nitochkina, and Yu.K.Orekin, JETF Lett., 16, (1972), 4

[3] Kondo K., T.Mashimoto, A.Sawaoka, and S.Saito, in. High Pressure Science and Technology, Proceedings of the 6th AIRAPT Conference, (1977) p.883

[4] Jamet F., and F.Bauer, in: Actes du Symposium International sur le Comportment des Milleux Denses sous Hauter Pressions Dynamiques, Editions du Comissariat a l'Energie Atomique, (1978) p.409

[5] Miller F., and E.Schulte, Z, fur Naturforschung, 33a, (1978) 918

[6] Zaretsky E.B., G.I.Kanel', P.A.Mogilevsky, V.E.Fortov, High Temperature Physics, 29, (1991) 1002

[7] Wark J.S., G.Klein, R.R.Whitlock, D.Riley, and N.C.Woolsey, J. Appl. Phys., 68, (1990) 4531

[8] Whitlock R.R., J.S.Wark, Phys. Rev. B, 52, (1995) 8

[9] Barker L.M. and Hollenbach R.E., Journ.Appl.Phys., 43, (1972) 4669

[10] Warren B.E., X-Ray Diffraction (Dower Publications Inc., New-York, 1990) p.85 